\title{
Experimental Studies on Plasmon Resonance of Ag Nanoparticles on Highly Ordered Pyrolytic Graphite (HOPG)
}

\author{
Ignacio Lopez-Salido, Nils Bertram, Dong Chan Lim, Gerd Ganteför, and Young Dok Kim ${ }^{\dagger, *}$ \\ Department of Physics, University of Konstanz, D-78457 Konstanz, Germany \\ ${ }^{\dagger}$ Division of Nano Sciences and Department of Chemistry, Ewha Womans University, Seoul 120-750, Korea \\ *E-mail:ydkim@ewha.ac.kr \\ Received January 11, 2006
}

\begin{abstract}
Studies on Ag nanoparticles grown on Highly Ordered Pyrolytic Graphite (HOPG) using HREELS provide different results for smaller and larger particle sizes corresponding to Ag coverages below and above 4 monolayers, respectively. For the larger particles, a positive frequency shift with decreasing particle size and a broadening of the plasmon resonance were observed with decreasing particle size, in line with previous studies on Ag on alumina. For the smaller particles, in contrast, a shift to lower energy with decreasing particle size, and a narrowing of the plasmon resonance with decreasing particle size can be found. The asymmetry of the Ag-features present for Ag coverages above 4 monolayers disappears for Ag coverages below 4 monolayers. The result for the smaller particles can be rationalized in terms of change of the particle growth mode with increasing particle size, which corroborates our STM data, as well as electronic effects due to the metal/support charge transfer.
\end{abstract}

Key Words : Ag, HOPG, Plasmon resonance, Electron spectroscopy

\section{Introduction}

As dimensions of particles decrease below several nanometers (nm), unusual electronic, geometric and chemical properties may appear: metals can become semiconductive, or semiconductors become metallic. ${ }^{1}$ Moreover, inert materials as bulk form can catalyze various reactions very efficiently, when particle size becomes below several nanometers. Behaviours of the nanoparticles can change as a result of interactions with their environments, e.g. catalytic activities of metal particles with a same size differ significantly upon changing support materials. ${ }^{2}$ Studies on properties of free and deposited nanoclusters and particles are not only of fundamental interest, but also related to various technologically important fields such as electronic devices, gas sensor, and catalysis. In particular, metal nanoparticles on Highly Ordered Pyrolytic Graphite (HOPG) surfaces show an interesting particle size selectivity in the chemical reactivity. ${ }^{3}$ Thus, investigations on electronic structures of $\mathrm{Ag}$ nanoparticles on the HOPG surfaces are related to the exploration of the origin of the size effect in heterogeneous catalysis. $^{3,4}$

To characterize electronic properties of metal particles, extensive studies on plasmon resonance have been carried out in the past using Electron Energy Loss Spectroscopy (EELS) as well as various optical methods. ${ }^{5,6}$ In particular, $\mathrm{Ag}$ has been widely investigated due to the distinct single plasmon resonance peak arising between 3-4 eV as well as its technological importance. ${ }^{5-20}$ Attention has been paid to the shifts of the plasmon resonance as a function of particle size, which can only be understood considering various factors at the same time. Changes of the particle shape as a function of size play a pivotal role, for example, a shift to lower binding energy with decreasing particle size was observed for Ag particles on titania as a result of the variations of the particle shape. ${ }^{7}$ Assuming that shapes of particles are constant, size dependent changes of electronic properties become important. On one hand, spill-out of selectrons on the surface decreases the surface electron density, leading to the negative shift of the surface plasmon frequency with respect to that of the bulk plasmon. Due to larger surface/volume ratios of smaller particles, such a shift to lower binding energy is expected with decreasing particle size. On the other hand, time-dependent density functional approach suggests that a shift of the plasmon frequency to higher binding energy with decreasing particle size is expected considering the mutual polarization of $\mathrm{s}$ and $\mathrm{d}$ electrons: in the core of particles, s electrons are significantly depolarized by $\mathrm{d}$ electrons. On the surface, in contrast, decrease of the s-d depolarizations due to the larger spill-out of the s electrons on the cluster surface increases the surface plasma frequency. ${ }^{8-13}$ When the particle density becomes larger, the coupling between particles also comes into play. ${ }^{5}$

Another important issue for the plasmon resonance is the peak broadening as a function of particle size. It is often observed that plasmon peaks become narrower with increasing particle size due to the increased lifetime of the collective oscillation, resulting from reduced electronsurface scattering mediated relaxations (1/R-Effect, R represents the radius of a particle). ${ }^{5,9,10,14,15}$

In this paper, the High Resolution EELS (HREELS) spectra taken for Ag particles grown by evaporation of $\mathrm{Ag}$ on mildly sputtered HOPG surfaces are presented. With increasing Ag coverage (measured in Monolayer Equivalents (MLE)), the average particle size increases gradually. At Ag 
coverages above 4 MLE, a shift to higher binding energy with decreasing particle size and a broadening of the plasmon resonance can be observed, in a good agreement with previous results. ${ }^{5,8-15}$ For lower $\mathrm{Ag}$ coverages, in contrast, a shift to lower binding energy and a narrowing of the plasmon resonance with decreasing particle size are found. In addition, the asymmetry of the Ag-features existing for metallic Ag particles disappears for $\mathrm{Ag}$ coverages below 4 MLE. The origin of the different results for $\mathrm{Ag}$ coverages below and above 4 MLE is discussed. It is suggested that geometric changes of the Ag nanoparticles as a function of particle size and different electronic structures for smaller particles compared to larger ones are closely related to the transition from positive to negative shift with decreasing particle size.

\section{Experimental Section}

Experiments were carried out in an Ultrahigh vacuum (UHV) system (base pressure $=1 \times 10^{-10}$ torr) equipped with HREELS (company: SPECS) and an ion gun for sputtering. The energy resolution of the spectrometer was $4 \mathrm{meV}$. The energy of primary electrons was $30 \mathrm{eV}$, and all spectra were collected in specula positions, allowing detection of only the non-zero dipole moment normal to the surface. Before HOPG samples were inserted into the UHV-chamber, the samples were cleaned by removing surface layers using scotch-tapes. Then, the samples were annealed at $1000 \mathrm{~K}$ in the UHV chamber. Before Ag was deposited on the surface, the HOPG samples were briefly sputtered (Acceleration energy $=0.5 \mathrm{kV}$, Ar pressure $=4 \times 10^{-5}$ torr, sample current $=3 \mu \mathrm{A}$, sputter time $=2$ seconds) in order to create nucleation centres of $\mathrm{Ag}$ atoms, and increase the stability of $\mathrm{Ag}$ particles. Ag was deposited with a constant $\mathrm{Ag}$ flux using a thermal metal evaporator, which was thoroughly outgassed before deposition. The Ag coverage on HOPG was measured using a quartz microbalance. The different sticking probabilities of Ag on HOPG and quartz were not taken into account, causing uncertainties for the determination of Ag coverages on HOPG. One should note that the sticking probability of $\mathrm{Ag}$ on HOPG can be as low as $10 \%$. The sample temperature was room temperature during $\mathrm{Ag}$ evaporation.

\section{Results and Discussion}

STM studies on Ag nanoparticles. We have recently performed Scanning Tunnelling Microscopy (STM) and Xray Photoelectron Spectroscopy (XPS) studies on Ag nanoparticles on HOPG prepared in the same way as those used for the HREELS studies in the present work. ${ }^{21}$ In Figure 1, a summary of our STM results for four different samples is shown. In the STM study, the particle height can be relatively accurately determined, whereas the particle diameter cannot be precisely estimated due to the additional broadening induced by the tip-particle convolution. We had measured the width of the half maximum of the particle

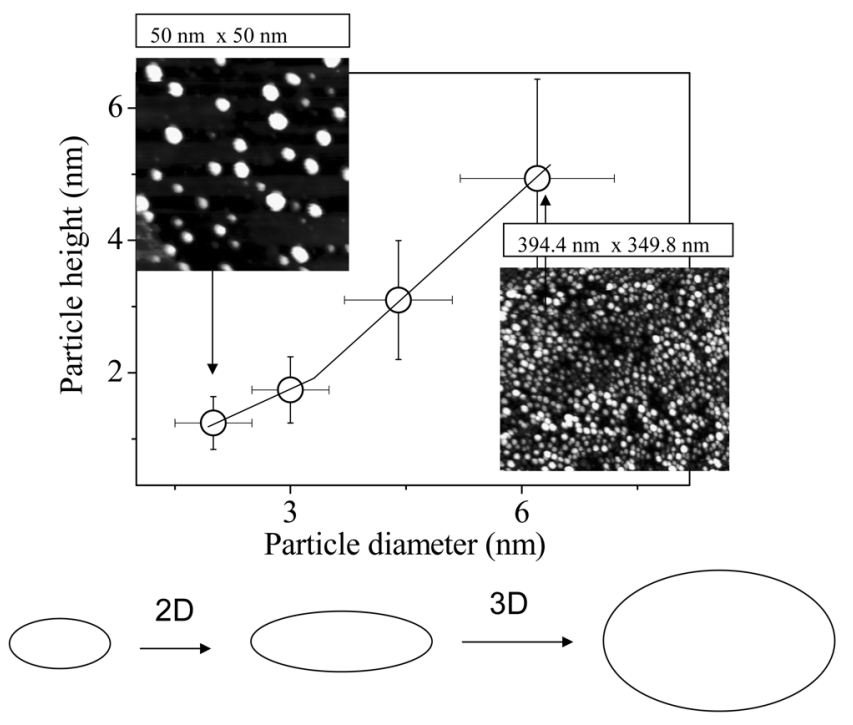

Figure 1. A summary of STM results on Ag nanoparticles on HOPG surfaces prepared in a similar way as those for the HREELS studies presented here. Four different samples were analyzed, and the average particle size of each sample is given in this plot. The particle densities of the samples for HREELS and STM studies can be different due to different sputtering time used for different samples. Deposition temperature was room temperature for all the samples. A simple sketch of the change of the particle growth mode is also illustrated.

profile, and then considered $60 \%$ of this value to be the real lateral size of a particle. The value " $60 \%$ " originates from our quantitative analysis of XPS spectra, as well as previous Transmission Electron Microscopy (TEM)/STM studies. ${ }^{21,22}$

First, it is obvious that the particle size increases with increasing $\mathrm{Ag}$ coverage, when the surface is sputtered equally, implying that an increased $\mathrm{Ag}$ coverage yields a larger average particle size on the HOPG surface. Second, the axial ratios (axis parallel to the surface/normal to the surface) of the particles are not constant, but it varies as a function of particle size.

2D-3D transition seems to takes place in the size range of 3-7 $\mathrm{nm}$ in diameter, and $2-4 \mathrm{~nm}$ in height, i.e. smaller particles grow more laterally than normal to the surface, whereas particle growth perpendicular to the surface becomes more facile for larger particles. The 2D-3D growth transition has already been found for metal particles on other substrates. ${ }^{1,23}$ We will show that the HREELS results can also be interpreted within this structural transition of $\mathrm{Ag}$ nanoparticles as a function of particle size. Previously, the axial ratio of the $\mathrm{Ag}$ nanoparticles grown on nanopits of HOPG was shown to be constant for all particle sizes (axial ratio $\sim 0.7) .{ }^{22}$ Our results are not completely in line with the previous results, yet one should take into account that the HOPG surfaces were treated differently in two different studies, and the Ag deposition temperatures were different. $^{21,22}$

HREELS studies (coverage above 4 MLE, 3D growth of $\mathbf{A g}$ ). In order to investigate electronic structures of $\mathrm{Ag}$ particles grown on HOPG, HREELS spectra were collected 

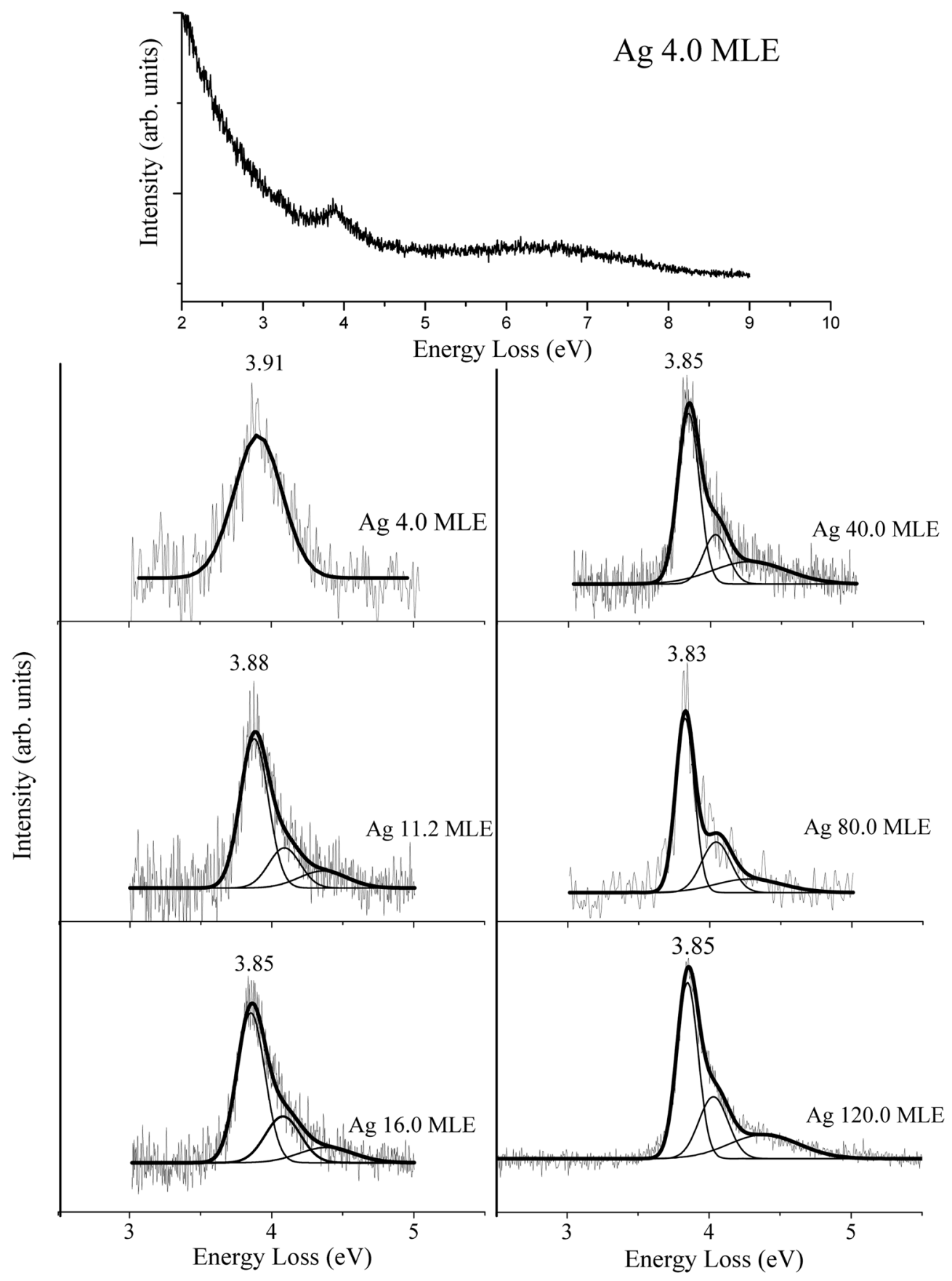

Figure 2. Series of HREELS spectra for Ag particles grown on mildly sputtered HOPG surfaces. (Ag coverages between 4 and 120 monolayers equivalent (MLE)). An exponentially decaying background was subtracted from each raw spectrum. A raw spectrum for a Ag coverage of 4.0 MLE in a energy range between $2-9 \mathrm{eV}$ is displayed on top of the figure. The Ag-derived features in this coverage regime can be fitted using combination of three Gaussian functions, which are attributed to the Ag-plasmon resonance, the interband (sp-d), and sp intraband transitions.

for the Ag coverage range from 0.8 MLE to 120 MLE (Fig. 2, 5,6). First, we focus on Ag coverages above 4 MLE (Fig. 2). With increasing $\mathrm{Ag}$ coverage, an asymmetric feature between 3.5 and $4.5 \mathrm{eV}$ can be observed, which is absent for a clean HOPG surface. Peaks at around $3.8 \mathrm{eV}$ can be attributed to the $\mathrm{Ag}$ plasmon resonance, whereas broad features at the higher energy regime originate from the interband transition between the $\mathrm{d}$ and $\mathrm{sp}$ bands (above $3.98 \mathrm{eV}$ ), and the electron-hole pair excitation within the $\mathrm{sp}$ band (above $3.86 \mathrm{eV}) .^{17,24}$

Plasmon resonance of nanoparticles can be explained within the Mie theory. According to the Mie theory, the surface resonance can be described by the following equation. $^{6}$

$$
\frac{\varpi_{p}}{\sqrt{\varepsilon_{m}+\varepsilon_{1}}}
$$


$\omega_{p}$ : bulk plasmon resonance which is about $9 \mathrm{eV}$ for $\mathrm{Ag}$ [6].

$\varepsilon_{m}$ : Dielectric constant for the matrix. 1 for vacuum.

$E_{1}$ : Dielectric constant for $\mathrm{Ag}$ which is $\sim 5 .^{25}$

The surface plasmon resonance of $\mathrm{Ag}$ is calculated to be $3.7 \mathrm{eV}$. When nanoparticles are deposited on other substrates, then the dielectric constant of the matrix changes, resulting in a shift of the plasmon resonance. For $\mathrm{Ag}$ nanoparticles on HOPG, we assume that $30 \%$ of the matrix consists of HOPG, and the rest $70 \%$ vacuum $\left(\varepsilon^{*}\right)$. The dielectric constant of HOPG is $\sim 2 .{ }^{26}$ One should also take into account that the particles are not spherical, but oblate, modifying the dielectric constant term. ${ }^{27}$ The depolarizaiton factor of the Ag particles originating from the non-spherical form can be estimated to be $0.3\left(\mathrm{~L}_{\perp}\right)$ normal to the surface (for the particles with diameters of 4-5 nm). One can roughly estimate the dielectric constant of the $\mathrm{Ag}$ nanoparticles on HOPG using the following equation.

$$
\varepsilon_{1}(\omega)=-\varepsilon_{m}^{*} \frac{1-L_{\perp}}{L_{\perp}}
$$

One gets the plasmon resonance of $3.2 \mathrm{eV}$ according to the equation above. We found that the $\mathrm{Ag}$ plasmon resonance is located between 3.8-3.9 eV, which is higher than the calculated values. An underestimation of $\mathrm{Ag}$ plasmon resonance by about $0.2 \mathrm{eV}$ was reported using the same calculations for Ag nanoparticles on alumina layers over $\mathrm{NiAl}$, which can be attributed to the strong interaction between the electron oscillation in the particle and the image dipole of the metallic substrate. ${ }^{10}$ The same explanation, however, only partially justifies the difference between the calculated and the measured resonance frequencies in our case, since the deviation between experiment and theory is larger than that of $\mathrm{Ag}$ on alumina. Probably, modified electronic structures of $\mathrm{Ag}$ nanoparticles compared to the bulk counterpart come into play for the plasmon resonance shifts, which could be dependent of substrate. We have recently performed XPS studies on $\mathrm{Ag} / \mathrm{HOPG}$, in which strong core level shifts of Ag nanoparticles compared to the bulk value were found. This was attributed to the initial state contribution, implying much different electronic structures of $\mathrm{Ag}$ nanoparticles compared to Ag bulk. ${ }^{21}$ In contrast, no initial state shifts of the $\mathrm{Ag}$ core levels were found for $\mathrm{Ag}$ on alumina. ${ }^{28}$ The origin of the positive core level shifts can be rehybridization of undercoordianted atoms as well as metal to support charge transfer. This issue is discussed more in detail elsewhere. ${ }^{29}$ Another possibility is that the contact area between $\mathrm{Ag}$ and carbon is much smaller than our assumption above.

With decreasing $\mathrm{Ag}$ coverage, the $\mathrm{Ag}$ plasmon feature becomes broader, and the peak position shifts to higher energies (blue shift). The FWHM (Full Width Half Maximum) of the Ag plasmon resonance for $\mathrm{Ag}$ coverages between 4 and 120 MLE vary from $0.35 \mathrm{eV}$ to $0.17 \mathrm{eV}$. In general, the plasmon resonance width was reported to be inversely proportional to the particle radius ( $1 / \mathrm{R}$-effect, $\mathrm{R}$ : Radius of particle) due to a lower electron-surface scattering rate for a larger particle, leading to an increased lifetime of the collective oscillation. ${ }^{5,9,10,14,15}$ The particle size distribution increases with increasing particle size, and therefore, the change of the plasmon resonance width cannot be justified by the heterogeneity of the particle size in a sample. ${ }^{20}$ Similar particle size dependent shifts of the plasmon frequency with decreasing particle size have been observed previously, and attributed to the size dependent screening efficiency of the Mie plasmon by d electrons as mentioned in the introduction.

In order to obtain better information on the size of $\mathrm{Ag}$ particles on HOPG, we collected ex-situ Atomic Force Microscopy (AFM) images for the samples prepared by the deposition of about 4 MLE of Ag on HOPG (Fig. 3). Detailed analysis of AFM images shows that the average height of the Ag particles on HOPG is about 2-4 nm, and the width is about $15-20 \mathrm{~nm}$. One has to consider that the ex-situ AFM studies are most likely influenced by contaminations (e.g. graphite layers on nanoparticles due to the decomposition of hydrocarbons) and therefore the actual particle size could be smaller than we have determined from the AFM images. In AFM, the height measurements can be reliable, whereas the determination of the particle width can include a large error due to the limited resolution caused by the width of the tip. Based on the STM results in Figure 1, the particle height of $2-4 \mathrm{~nm}$ corresponds to the lateral size
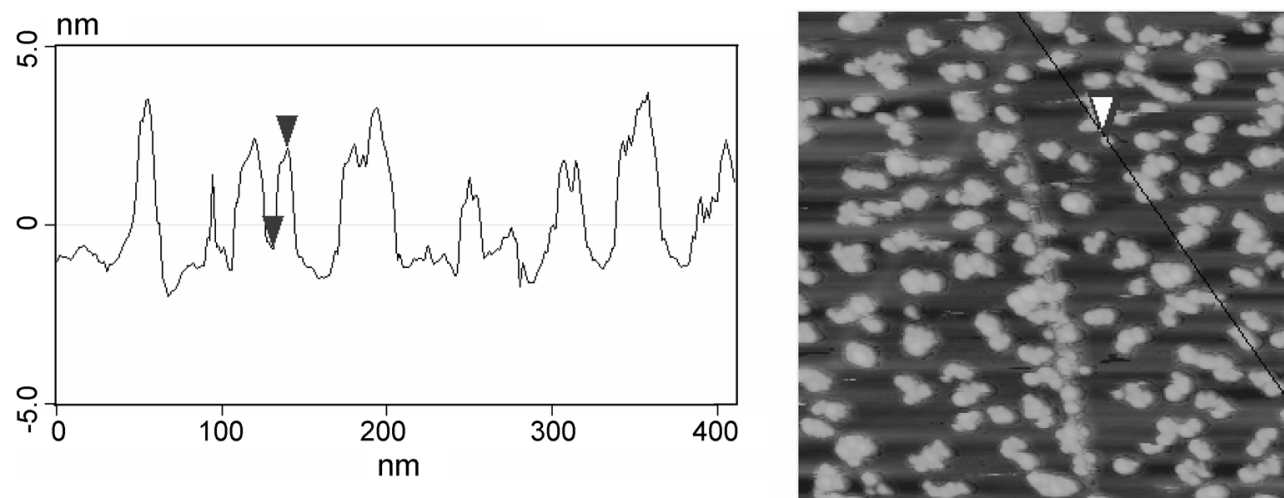

Figure 3. AFM image and a height profile of Ag particles on HOPG. About 4 MLE of Ag was deposited on HOPG. 

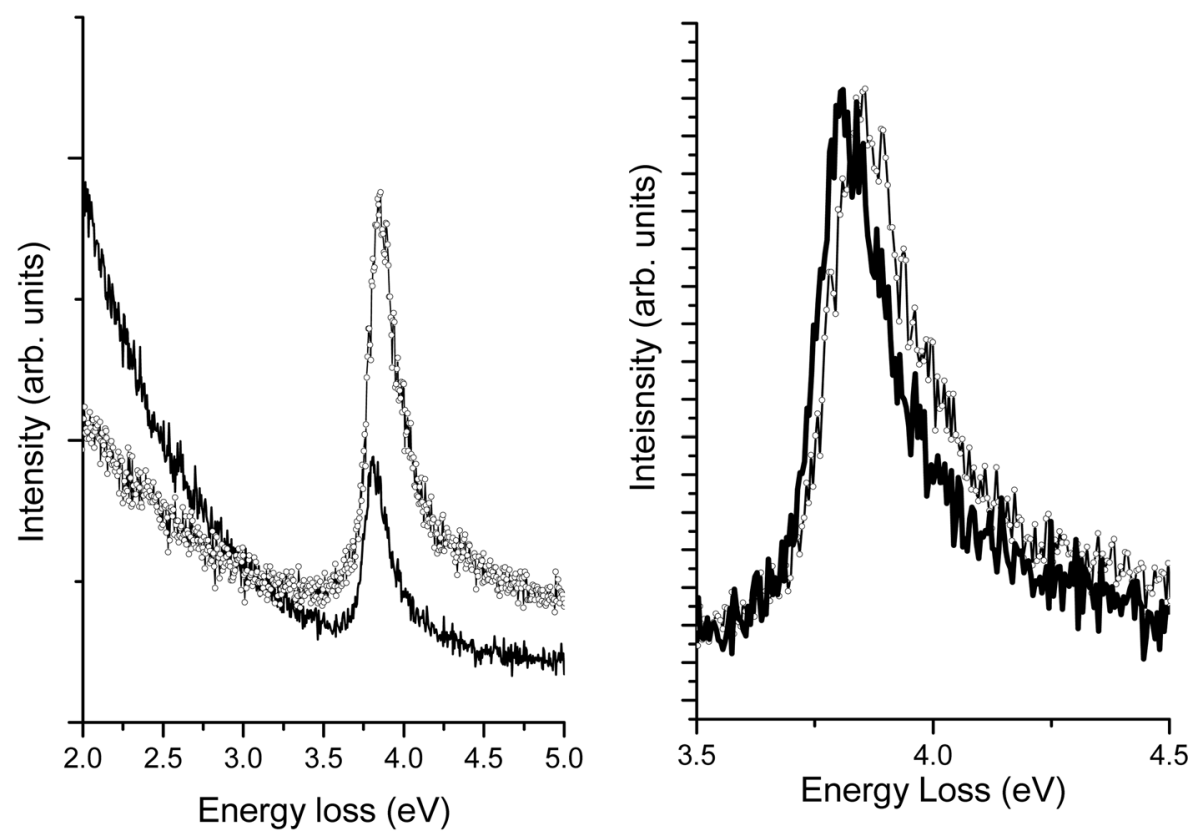

Figure 4. HREELS spectra of Ag on HOPG before (dot + line) and after heating (line) to about $400 \mathrm{~K}$. A narrowing of the peak width and a shift to lower binding energy is observed upon heating the sample to $400 \mathrm{~K}$. Left: raw data Right: After subtracting background and normalization.

of 3-6 $\mathrm{nm}$, i.e. the HREELS results presented in this section are related to the particle diameters above 3-6 $\mathrm{nm}$. In this size regime, the particle growth normal to the surface is as fast as (or even faster than) lateral growth, as mentioned above (Fig. 1), and therefore, the HREELS spectra shown in this section correspond to the $3 \mathrm{D}$ growth regime. The axial ratio of the $\mathrm{Ag}$ particles in this coverage range increases with increasing particle size, and this could also partially contribute to the plasmon resonance shifts.

We collected HREELS spectra for the Ag covered HOPG surface (Ag coverage: $120 \mathrm{MLE}$ ) before and after heating to about $400 \mathrm{~K}$ (Fig. 4). After heating to about $400 \mathrm{~K}$, the Ag plasmon peak intensity is reduced, whereas the HOPG features have grown compared to the spectrum taken before heating. These results can indicate that the particles on HOPG agglomerate upon heating to form larger particles. An alternative interpretation is the improvement of the crystallinity of the Ag nanoparticles (of film in this case) after annealing. We rule out the possibility for the evaporation of $\mathrm{Ag}$ upon heating, since $400 \mathrm{~K}$ is much lower than the evaporation temperature of Ag. After heating the sample, the Ag plasmon peak becomes narrower (FWHM decreases by $0.04 \mathrm{eV}$ upon) and the plasmon peak shifts to the lower binding energy by $0.02 \mathrm{eV}$.

HREELS studies (coverage below 4 MLE, 2D growth of Ag). In Figure 5, HREELS spectra taken for Ag coverages below 4 MLE are illustrated. Based on the STM and AFM data, one can conclude that the Ag coverages below 4 MLE corresponds to the particle size range, in which the particles grow more laterally than normal to the substrate surface (quasi-2D growth), resulting in an increase of the axial ratio of the particles with decreasing particle size. In the regime of the quasi-2D growth of $\mathrm{Ag}$ nannoparticles, the plasmon resonance perpendicular to the surface should shift to lower frequencies with decreasing particle size according to the equation 2. In fact, a plasmon frequency shift to lower energy is observed with decreasing particle size, which is different from the results for Ag coverages above 4 MLE. The best fits of these peaks can be achieved using a single Gaussian function, i.e. the asymmetry of the peak observed for the larger $\mathrm{Ag}$ coverages disappears for $\mathrm{Ag}$ coverages below 4 MLE.

Besides the geometric factor of the particles, the electronic factor should be also considered to understand the origin of the plasmon frequency shifts as a function of $\mathrm{Ag}$ particle size. For the larger particles, the influence of the charge transfer from/to supports might be negligible, due to the increased screening of the additional charges by neighboring metal atoms. For the smaller particles, in contrast, electronic properties of Ag particles can significantly be modified by charge transfers between Ag particles and sputtered-HOPG, resulting in deviations from intrinsic particle size effects on the plasmon resonance width. Considering that the work function of carbon and HOPG surfaces are $\sim 4.6 \mathrm{eV}$, and that of $\mathrm{Ag}$ around $4.2 \mathrm{eV}$, small $\mathrm{Ag}$ particles should be positively charged (if there is any charging). ${ }^{21,31,32}$ In the previous XPS studies, the binding energies of $3 \mathrm{~d}, 4 \mathrm{~d}$ and $5 \mathrm{sp}$ electrons of Ag on carbon substrates become larger for smaller particles compared to the bulk, which was recently attributed to the initial state effects of photoemission by the Auger analysis by some authors of the present work. ${ }^{21,29,33}$ Taking into account that we recently found much smaller core level shifts for $\mathrm{Au}$ (work function $>5 \mathrm{eV}$ ) nanoparticles on HOPG comparing to the case of $\mathrm{Ag}$, the positive core level shifts of $\mathrm{Ag} \mathrm{3d}, 4 \mathrm{~d}$ and $5 \mathrm{sp}$ levels for the Ag nanoparticles could be related to the partial positive charging of the Ag particles. ${ }^{29}$ 


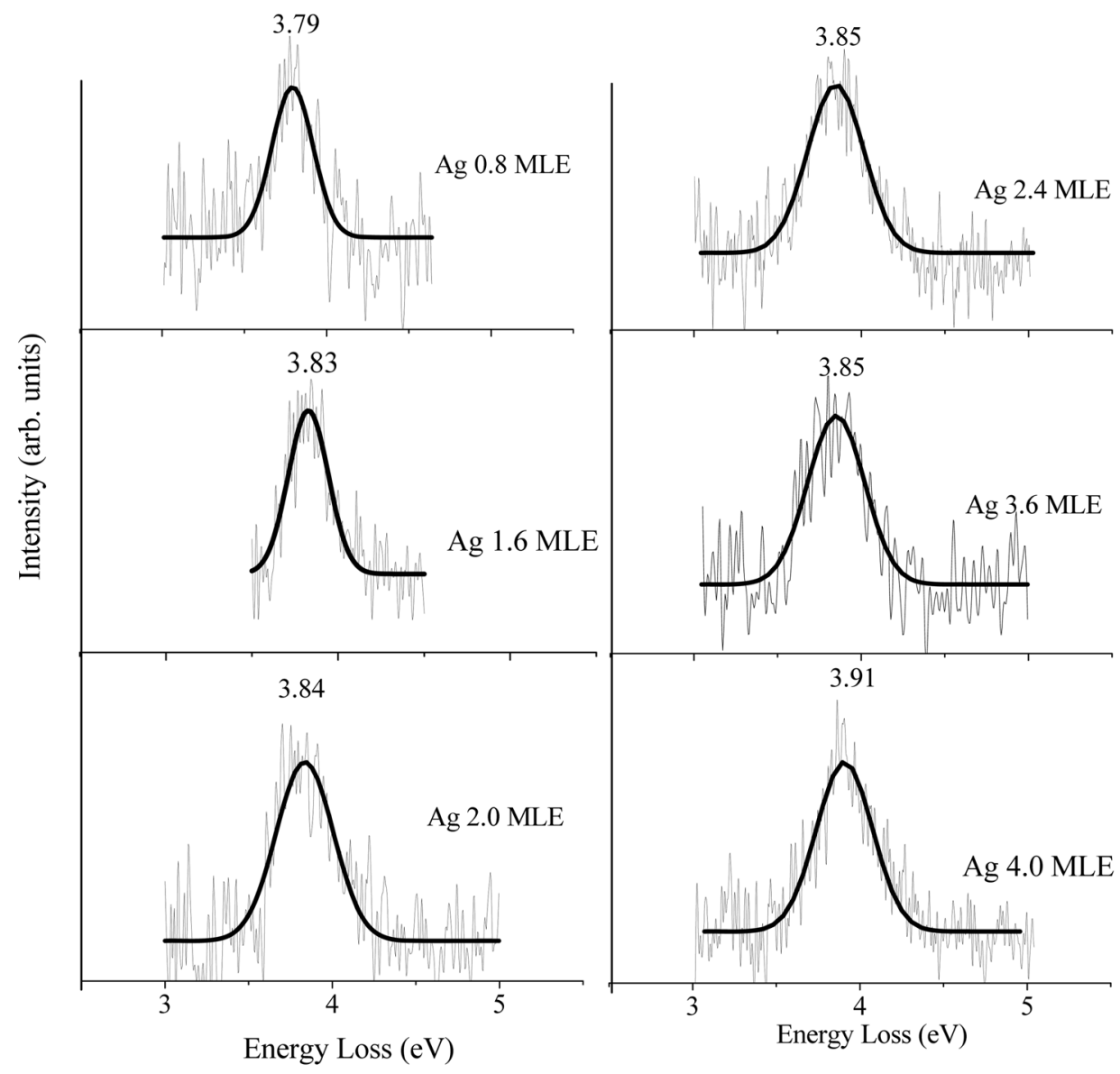

Figure 5. HREELS spectra for Ag particles grown on mildly sputtered HOPG surfaces. (Ag coverages below 4 monolayers equivalent (MLE)) An exponentially decaying background was subtracted from each spectrum. For these spectra, the best fits can be achieved using a single Gaussian function, indicating the disappearance of the interband and intraband transition with decreasing particle size

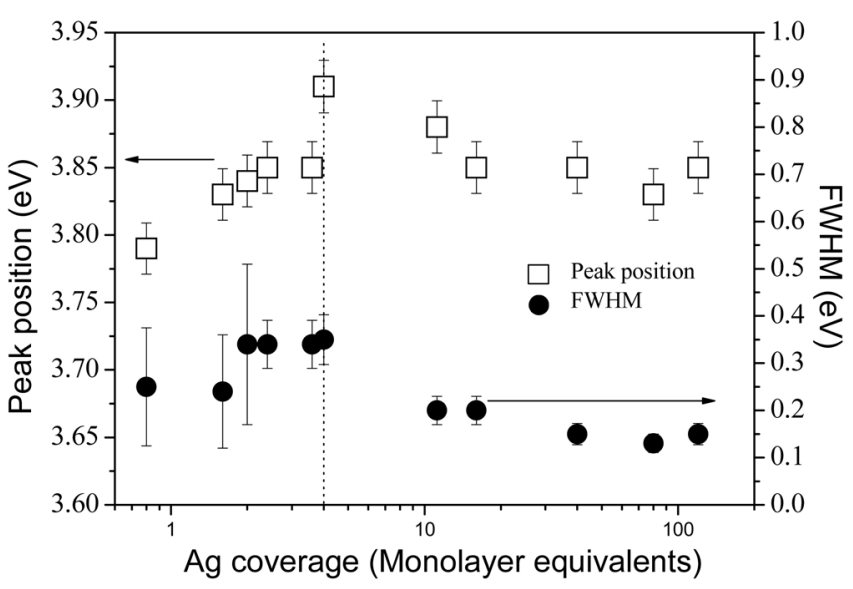

Figure 6. Variations of the peak position and width for the $\mathrm{Ag}$ plasmon resonance as a function of Ag coverage.

When $5 \mathrm{~s}$ electrons are partially transferred to carbon, the $5 \mathrm{~s}$ electron density decreases, yielding a shift of the plasmon frequency to lower energy. Further theoretical studies may be required to shed light on the possible plasmon frequency shifts caused by particle charging. It is important to mention that $\mathrm{Ag}$ nanoparticles in a reactive matrix such as $\mathrm{CO}$ show a red-blue shift transition (frequency shift first to higher bind- ing energy and then lower binding energy with decreasing particle size) analogous to our results, implying that here a carbon substrate may act like $\mathrm{CO}$ matrix in modifying electronic structures of Ag. ${ }^{9}$

The plasmon peak width decreases with decreasing Ag coverage (or decreasing particle size), which does not match with the $1 / \mathrm{R}$-trend observed for larger particles. It is in principle possible that the metal-support interactions can be considered to rationalize the deviation from the $1 / \mathrm{R}$-effect on the plasmon resonance width. In colloidal solution, the charge transfer from or to particles can significantly change the plasmon resonance width. ${ }^{29}$ However, in previous studies, a chemical reaction between matrix and Ag particles caused a broadening of the plasmon resonance width, which does not reconcile our data. ${ }^{9,30}$ Therefore, the narrowing of the resonance line with decreasing particle size is rather related to different particle size distributions for different samples than the Ag-carbon interactions. ${ }^{21}$

It is important to mention that the shift to lower energy of the Ag plasmon frequency with decreasing particle size was not observed in the similar particle size range for $\mathrm{Ag}$ on alumina thin films, in which the axial ratios of particles were almost constant as a function of size. This result suggests that the HREELS data are quite sensitive to the geometric 
and electronic structures of nanoparticles, and therefore, can be used to better understand the structural properties of metal nanoparticles on the surface.

\section{Summary}

In conclusion, HREELS spectra taken for Ag nanoparticles on HOPG are presented for Ag coverages between 0.8 and 120 MLE. As we have shown using STM, these changes of plasmon resonances as a function of $\mathrm{Ag}$ coverage are related to the different mean particle sizes at different coverages. Above 4 MLE, a broadening and a positive shift of plasmon frequency with decreasing particle size were detected, which are in a good agreement with previous results, and explained mainly in terms of screening of the free electrons due to the depolarization field of $\mathrm{d}$ electrons, which is lowered for electrons of cluster surfaces. In addition, structural changes of particles should be also taken into account. A negative shift of the plasmon resonance frequency with decreasing particle size and a deviation from the $1 / \mathrm{R}$-effect for the width of the plasmon resonance were observed for the $\mathrm{Ag}$ coverage below $4 \mathrm{MLE}$, which is completely different from the results for Ag coverages above 4 MLE. The negative plasmon frequency shift with decreasing particle size can be attributed to either the quasi-2D growth of metal particles in this coverage range, or partial charge transfer between $\mathrm{Ag}$ and carbon. We demonstrate that HREELS studies on deposited metal particles can open new insights into the electronic structures, and metal-support interactions of small nanoparticles.

We acknowledge DFG (Deutsche Forschungsgemeinschaft) for the financial support.

\section{References}

1. Valden, M.; Lai, X.; Goodman, D.W. Science 1998, 281, 1647.

2. Haruta, M.; Tsubota, S.; Kobayashi, T.; Kageyama, H.; Genet, M. J.; Delmon, B. J. J. Catal. 1993, 144, 175.

3. Lim, D. C.; Lopez-Salido, I.; Kim, Y. D. Surf. Sci. 2005, 598, 96.
4. Bukhtiyarov, V. I.; Carley, A. F.; Dollar, L. A.; Roberts, M. W. Surf. Sci. Lett. 1997, 381, L605.

5. Kreibig, W.; Vollmer, M. Optical Properties of Metal Clusters, Springer Series in Material Science 25; Springer: 1995.

6. Rocca, M. Surf. Sci. Rep. 1995, 22, 1.

7. Wenzel, T.; Bosbach, J.; Stietz, F.; Träger, F. Surf. Sci. 1999, 432, 257.

8. Liebsch, A. Phys. Rev. B 1993, 48, 11317.

9. Kreibig, U.; Genzel, L. Surf. Sci. 1985, 156, 678.

10. Nilius, N.; Ernst, N.; Freund, H.-J. Phys. Rev. Lett. 2000, 84, 3994.

11. Eckardt, W.; Tran Thoai, D. B.; Frank, F.; Schulze, W. Solid State Comm. 1983, 46, 571

12. Charle, K.-P.; Schulze, W.; Winter, B. Z. Phys. D 1989, 12, 471.

13. Tiggesbäumer, J.; Köller, L.; Meiwes-Broer, K.-H.; Liebsch, A. Phys. Rev. A 1993, 48, R1749.

14. Genzel, L.; Martin, T. P.; Kreibig, U. Z. Phys. B 1975, 21, 339.

15. Hövel, H.; Fritz, S.; Hilger, A.; Kreibig, U.; Vollmer, M. Phys. Rev. B 1993, 48, 18178.

16. Savio, L.; Vattuone, L.; Rocca, M. Phys. Rev. B 2003, 67, 045406.

17. Grimaud, C.-M.; Siller, L.; Andersson, M.; Palmer, R. E. Phys. Rev. B 1999, 59, 9874.

18. Harbich, W.; Fedrige, S.; Buttet, J. Z. Phys. D 1993, 26, 138.

19. Harbich, W.; Fedrigo, S.; Buttet, J. Chem. Phys. Lett. 1992, 195, 613.

20. Schaffner, M.-H.; Patthey, F.; Schneider,W.-D. Surf. Sci. 1998, $417,159$.

21. Lopez-Salido, I.; Lim, D. C.; Kim, Y. D. Surf. Sci. 2005, 588, 6.

22. Hövel, K.; Becker, Th.; Nettac, A.; Reihl, B.; Tschudy, M.; Williams, E. J. J. Appl. Phys. 1997, 81, 154.

23. Campbell, C. T. Surf. Sci. Rep. 1997, 27, 1.

24. Winsemius, P.; Kampen, F. F.; Lengbeeck, H. P.; Van Went, G. G. J. Phys. F 1976, 6, 1583.

25. Ehrenreich, E.; Philipp, H. Phys. Rev. 1962, 128, 1622.

26. Djurisic, A. B.; Li, E. H. J. Appl. Phys. 1999, 85, 7404.

27. Gans, R. Ann. Phys. 1912, 37, 881.

28. Luo, K.; Lai, X.; Yi, C.-Y.; Davis, A.; Gath, K. K.; Goodman, D. W. J. Phys. Chem. B 2004, 109, 4064.

29. Lopez-Salido, I.; Lim, D. C.; Kim, Y. D. J. Phys. Chem. B 2006, 110,1128

30. Linnert, T.; Mulvaney, P.; Hengle, A. J. Phys. Chem. 1993, 97, 679.

31. Suzuki, S.; Bower, C.; Watanabe, Y.; Zhou, O. Appl. Phys. Lett. 2000, 76, 4007.

32. Kim, Y. D.; Wei, T.; Wendt, S.; Goodman, D. W. Langmuir 2003, 19, 7929.

33. Wertheim, G. K.; DiCenzo, S. B.; Buchanan, D. N. E. Phys. Rev. B 1986, 33, 5384 . 